\title{
Choice of laxatives and colonoscopic preparation in pregnant patients from the viewpoint of obstetricians and gastroenterologists
}

\author{
Jeevan Vinod, Jennifer Bonheur, Burton I Korelitz, Georgia Panagopoulos
}

\author{
Jeevan Vinod, Jennifer Bonheur, Burton I Korelitz, Georgia \\ Panagopoulos, Department of Gastroenterology, Lenox Hill \\ Hospital, New York University School of Medicine, New York \\ 10021, United States \\ Correspondence to: Burton I Korelitz, MD, Department of \\ Gastroenterology, Lenox Hill Hospital, and New York University \\ School of Medicine, 100 East $77^{\text {th }}$ Street, New York, NY 10021, 6 \\ Black Hall, New York, United States. bkorelitz@1enoxhill.net \\ Telephone: +1-212-4342000 \\ Received: August 29, 2007 Revised: October 11, 2007
}

\begin{abstract}
AIM: To elucidate the preferences of gastroenterologists at our institution and compare them to those of obstetricians when making decisions in the pregnant patient, including which type of bowel preparations to use for flexible sigmoidoscopy or colonoscopy, as well as which laxatives can be used safely.
\end{abstract}

METHODS: Surveys were mailed to all attending gastroenterologists $(n=53)$ and obstetricians $(n=99)$ at our institution. Each survey consisted of the 14 most common laxative or motility agents used in pregnancy and inquired about the physician's prescribing habits in the past as well as their willingness to prescribe each medication in the future. The survey also listed four common bowel preparations used prior to colonoscopy and sigmoidoscopy and asked the physician to rank the order of the preferred agent in each case.

RESULTS: With regard to common laxatives, both gastroenterologists and obstetricians favor the use of Metamucil, Colace, and Citrucel. Both groups appear to refrain from using Fleets Phosphosoda and Castor oil. Of note, obstetricians are less inclined to use PEG solution and Miralax, which is not the case with gastroenterologists. In terms of comparing bowel preparations for colonoscopy, $50 \%$ of gastroenterologists prefer to use PEG solution and $50 \%$ avoid the use of Fleets Phosphosoda. Obstetricians seem to prefer Fleets Phosphosoda (20\%) and tend to avoid the use of PEG solution (26\%). With regard to bowel preparation for sigmoidoscopy, both groups prefer Fleets enema the most $(51 \%)$, while magnesium citrate is used least often (38\%).

CONCLUSION: It is clear that preferences in the use of bowel cleansing preparations between the two groups exist, but there have not been many case controlled human studies in the pregnant patient that give clear cut indications for using one versus another drug. In light of the challenge of performing controlled trials in pregnant women, more extensive surveys should be undertaken to gather a larger amount of data on physicians' experiences and individual preferences.

\section{(c) 2007 WJG. All rights reserved.}

Key words: Laxative; Pregnancy; Colonoscopy; Sigmoidoscopy; Gastroenterologists; Obstetricians

Vinod J, Bonheur J, Korelitz BI, Panagopoulos G. Choice of laxatives and colonoscopic preparation in pregnant patients from the viewpoint of obstetricians and gastroenterologists. World J Gastroenterol 2007; 13(48): 6549-6552

http://www.wjgnet.com/1007-9327/13/6549.asp

\section{INTRODUCTION}

There is a degree of apprehension when it comes to altering medical care in pregnant female patients. On some occasions pregnant women will require colonoscopic evaluation and the clinician will be faced with a decision on what type of bowel preparation to use for flexible sigmoidoscopy or colonoscopy. Also, constipation is a common problem faced by many women during the course of pregnancy and a choice of laxatives must be made by the physician. Given the numerous pharmacological choices available and the lack of data in the medical literature, these decisions are not always easy to make.

When considering endoscopy in the pregnant patient, the physician must balance the safety concerns of both mother and fetus against the relative value of the information obtained or the diagnostic procedure performed ${ }^{[1]}$. All available evidence suggests that sigmoidoscopy is safe during pregnancy and the indications include rectal bleeding, chronic diarrhea, abdominal pain, and rectal pain ${ }^{[2]}$. Guidelines for colonoscopy in pregnancy are not readily available due to insufficient data, although studies which have been done demonstrate safety and efficacy of the procedure provided that obstetrical consultation and close monitoring take place. Colonoscopy 
is indicated for suspected colon cancer, uncontrolled severe hemorrhage, or when necessary before colonic surgery ${ }^{[3]}$ in pregnant women as well as the general population.

After the indications for an endoscopic procedure are established, the next question becomes which bowel preparation to use. Developing guidelines for this poses unique challenges since there are few controlled trials that include pregnant patients. However, there is some information available regarding the efficacy of agents such as tap water enemas, castor oil, polyethylene glycol (PEG) solution, magnesium citrate, senna, bisacodyl, docusate, and phosphosoda ${ }^{[4,5]}$. The purpose of this study was to elucidate the preferences of gastroenterologists at our institution and compare them to those of obstetricians when making such decisions.

\section{MATERIALS AND METHODS}

After obtaining IRB approval, surveys were mailed to all attending gastroenterologists $(n=53)$ and obstetricians $(n=99)$ at our institution. Each survey consisted of the 14 most common laxative or motility agents used in pregnancy and inquired about the physician's prescribing habits in the past as well as their willingness to prescribe each medication in the future. The survey also listed four common bowel preparations used prior to colonoscopy and sigmoidoscopy and asked the physician to rank the order of the preferred agent in each case. Descriptive statistics were computed using SPSS Version 14.0. (SPSS Inc. Chicago, Illinois). Valid percentages were used in order to account for those surveys that were incomplete. Utilizing Fisher's Exact Test, the proportion of gastroenterologists who would prescribe the listed laxatives was compared to the proportion of obstetricians who also indicated they would prescribe. $P<0.5$ was a priori considered to indicate statistical significance.

\section{RESULTS}

Seventy five surveys were returned making the total response rate nearly $50 \%$ (53\% of gastroenterologists and $47 \%$ of obstetricians). Among gastroenterologists, $96 \%$ of respondents were male and $4 \%$ female, while $55 \%$ of obstetricians were male and $45 \%$ female. The mean number of years in practice between both groups was 19 \pm 11 years and the mean physician age was $41 \pm 22$ years.

With regard to the common laxatives, the overwhelming majority of gastroenterologists were inclined to prescribe Metamucil (psyllium), Citrucel (methylcellulose), and Colace (docusate). Some had a preference for using glycerin suppositories, Fleets enemas (sodium phosphate enemas), tap water enemas, and Miralax (PEG 3350), although not as often as the agents above. Meanwhile, most gastroenterologists are reluctant to prescribe Fleets Phosphosoda (oral sodium phosphate), castor oil, and oral Dulcolax (bisacodyl). Table 1 presents the exact percentages as described above.

The vast majority of obstetricians favor the use of Colace, Metamucil, and glycerin suppositories. They also prefer to use Citrucel, Fleets enemas, Dulcolax suppositories, and tap water enemas, although not as often as those listed above. The majority of obstetricians are less inclined to use PEG solution, Mineral oil, and Fleets Phosphosoda. Table 1 presents the exact percentages as described above.

As seen in Table 1, it is evident that both gastroenterologists and obstetricians favor the use of Metamucil $(P=1.00)$, Colace $(P=0.293)$, and Citrucel $(P=0.164)$. Both groups appear to refrain from using Fleets phosphosoda $(P=0.051)$ and Castor oil $(P=0.402)$. Of note, obstetricians are less inclined to use PEG solution $(P=0.001)$, Miralax $(P=0.000)$, and tap water enema $(P=0.046)$, which is not the case with gastroenterologists.

In terms of comparing bowel preparations for colonoscopy, it is apparent that $50 \%$ of gastroenterologists prefer to use PEG solution and $50 \%$ avoid the use of Fleets Phosphosoda. Obstetricians seem to prefer Fleets Phosphosoda (20\%) and tend to avoid the use of PEG solution (26\%), which is the exact opposite as gastroenterologists. With regard to bowel preparation for sigmoidoscopy, the preferences between gastroenterologists and obstetricians appear to be quite similar. Both groups prefer Fleets enema the most (51\%), while magnesium citrate is used least often $(38 \%)$.

\section{DISCUSSION}

Several conclusions can be drawn from the results of this physician survey. Both groups commonly prescribe Metamucil, Citrucel, and Colace. All are frequently used bulk-producing medications that carry low pregnancy risk factors and are without reported complications ${ }^{[6]}$. The overall frequency of PEG solution being used is much higher with gastroenterologists. Studies have shown that the systemic absorption of PEG is minimal and the problems with abdominal bloating and gas are less common as compared to other osmotic laxatives ${ }^{[5]}$. Another similarity common to both groups is the avoidance of phosphosoda preparations. This may be related to the fact that in published studies, newborns were shown to manifest bone demineralization and bone growth failure because of maternal phosphate overload $^{[10]}$, although a one time use in pregnancy has not shown to be detrimental. Another consideration when using phosphosoda preparations is the risk of phosphate nephropathy ${ }^{[12]}$, which has been reported in select cases.

The overall majority of the available laxatives are without documented side effects; however castor oil carries an absolute contraindication for use in pregnancy (risk factor $\mathrm{X}$ ) because it has been linked to uterine rupture ${ }^{[7]}$. Mineral Oil has also been associated with adverse effects during pregnancy and should be avoided because it can impair maternal fat soluble vitamin absorption, leading to neonatal coagulopathy and hemorrhage ${ }^{[8]}$. From the results above it appears to be generally avoided in both groups, but significantly more so among obstetricians. Another apparent difference is the prescribing of tap water enemas in both groups. Obstetricians seem more reluctant to prescribe it perhaps due to the possible induction of labor 
Table 1 Comparison of gastroenterologists' (GI) vs obstetricians' (OB) preference of 14 common laxative/motility agents prescribed in the pregnant patient

\begin{tabular}{|c|c|c|c|c|c|}
\hline & $\begin{array}{l}\% \text { of GI physicians who have } \\
\text { prescribed in the past or } \\
\text { would prescribe in the future }\end{array}$ & $\begin{array}{l}\% \text { of OB physicians who } \\
\text { have prescribed in the past } \\
\text { or would prescribe in future }\end{array}$ & $\begin{array}{c}\% \text { of GI physicians } \\
\text { who would NOT } \\
\text { prescribe }\end{array}$ & $\begin{array}{c}\% \text { of OB physicians } \\
\text { who would NOT } \\
\text { prescribe }\end{array}$ & $\underset{\text { value }}{\boldsymbol{P}}$ \\
\hline Metamucil (psyllium) & 96.5 & 95.6 & 3.9 & 2.2 & 1.000 \\
\hline Citrucel (methylcellulose) & 92.9 & 77.1 & 7.1 & 11.4 & 0.164 \\
\hline Colace (docusate) & 89.3 & 97.7 & 10.7 & 2.3 & 0.293 \\
\hline Glycerin suppository & 77.8 & 82.0 & 14.8 & 15.4 & 0.757 \\
\hline Fleets enema (sodium phosphate enema) & 75.0 & 55.0 & 25.0 & 41.7 & 0.174 \\
\hline Tap water enema & 70.4 & 44.5 & 22.2 & 50.0 & 0.046 \\
\hline Miralax (PEG 3350) & 69.2 & 20.6 & 23.1 & 51.7 & 0.000 \\
\hline Magnesium Citrate & 54.2 & 32.3 & 45.8 & 61.8 & 0.113 \\
\hline GoLytely (PEG solution) & 53.8 & 12.9 & 46.2 & 80.6 & 0.001 \\
\hline Dulcolax (bisacodyl suppository) & 50.0 & 45.8 & 34.6 & 54.3 & 0.799 \\
\hline Mineral Oil & 46.2 & 25.0 & 42.3 & 75.0 & 0.106 \\
\hline Dulcolax (bisacodyl oral) & 30.7 & 38.3 & 53.8 & 52.9 & 0.595 \\
\hline Castor Oil & 26.1 & 37.6 & 60.9 & 62.5 & 0.402 \\
\hline Fleets Phosphosoda (oral sodium phosphate) & 7.7 & 29.1 & 92.3 & 71.0 & 0.051 \\
\hline
\end{tabular}

with the use of a rectal enema, although it has been shown to be quite safe in the pregnant patient ${ }^{[3]}$.

Another explanation for the differences between the two groups may also be due to preexisting medical conditions in the pregnant patient such as Crohn's disease, which is exacerbated by various medications seen more frequently by gastroenterologists. Such exacerbations may adversely affect the pregnancy ${ }^{[1]}$, although there is no established link with the 14 common laxatives to Crohn's disease exacerbation.

Regarding preparation for colonoscopy, gastroenterologists favor PEG solution, while there appears to be more hesitation among obstetricians. It is unclear whether this is because gastroenterologists have greater experience in its use or simply because data is lacking regarding the safety of medications for colonoscopy and pregnancy. For flexible sigmoidoscopies, both groups favor Fleets enemas, although it has been shown that tap water enemas appear to be the safest option, even if not as effective, particularly during the first trimester ${ }^{[9]}$.

In conclusion, it is clear that preferences in the use of bowel cleansing preparations between the two groups exist, but there have not been many case controlled human studies in the pregnant patient that give clear cut indications for using one versus another drug. Therefore, it seems logical that both groups favor medications that seem to respond best with the majority of patients without causing harm or compromising the pregnancy. In light of the challenge of performing controlled trials in pregnant women, more extensive surveys should be undertaken to gather a larger amount of data on physicians' experiences and individual preferences. Perhaps this will provide a clearer understanding of which laxatives and purgatives are optimal to use during pregnancy.

\section{COMMENTS}

\section{Background}

Prescribing medications to the pregnant patient can be challenging. The purpose of the research study was to gain an understanding of the different practices used by obstetricians and gastroenterologists when prescribing laxatives and using bowel preparations for colonoscopy and sigmoidoscopy.

\section{Research frontiers}

There have not been many case controlled human studies in the pregnant patient, therefore surveys were used to gather data on physician prescribing habits.

\section{Innovations and breakthroughs}

Due to limited research in the pregnant patient, the majority of articles point toward individual physician preference and patient response when prescribing a particular medication.

\section{Applications}

In light of the challenge of performing controlled trials in the pregnant patient, more extensive surveys should be undertaken to gather a larger amount of data on physicians' experiences and individual preferences.

\section{Peer review}

This paper is well written and covers an important clinical area. This is an interesting manuscript on the preference of laxatives and colonoscopic preparation in pregnancy of obstetricians and gastroenterologists.

\section{REFERENCES}

1 Katz JA. Endoscopy in the pregnant patient with inflammatory bowel disease. Gastrointest Endosc Clin N Am 2002; 12: 635-646

2 Cappell MS, Sidhom O. Multicenter, multiyear study of safety and efficacy of flexible sigmoidoscopy during pregnancy in 24 females with follow-up of fetal outcome. Dig Dis Sci 1995; 40: 472-479

3 Cappell MS. The fetal safety and clinical efficacy of gastrointestinal endoscopy during pregnancy. Gastroenterol Clin North Am 2003; 32: 123-179

4 Mahadevan U, Kane S. American gastroenterological association institute technical review on the use of gastrointestinal medications in pregnancy. Gastroenterology 2006; 131: 283-311

5 Prather CM. Pregnancy-related constipation. Curr Gastroenterol Rep 2004; 6: 402-404

6 Bonapace ES Jr, Fisher RS. Constipation and diarrhea in pregnancy. Gastroenterol Clin North Am 1998; 27: 197-211

7 Sicuranza GB, Figueroa R. Uterine rupture associated with castor oil ingestion. J Matern Fetal Neonatal Med 2003; 13: 133-134

8 Hasler WL. The irritable bowel syndrome during pregnancy. Gastroenterol Clin North Am 2003; 32: 385-406, viii

9 Cappell MS. The safety and efficacy of gastrointestinal endoscopy during pregnancy. Gastroenterol Clin North Am 1998. 
27: $37-71$

10 Rimensberger $\mathbf{P}$, Schubiger $\mathrm{G}$, Willi U. Connatal rickets following repeated administration of phosphate enemas in pregnancy: a case report. Eur J Pediatr 1992; 151: 54-56

11 Baiocco PJ, Korelitz BI. The influence of inflammatory bowel disease and its treatment on pregnancy and fetal outcome. $J$ Clin Gastroenterol 1984; 6: 211-216

12 Desmeules S, Bergeron MJ, Isenring P. Acute phosphate nephropathy and renal failure. N Engl J Med 2003; 349: 1006-1007

S- Editor Zhu LH L- Editor Alpini GD

E- Editor Liu Y 